effects on IQ and genetic and environmental factors must be considered among possible causes of behavioral problems associated with early unilateral brain damage. Children with diffuse brain damage are at higher risk of behavioral and cognitive dysfunction than those with focal lesions.

\title{
STIMULANT AND SSRI MEDICATION TRENDS IN ADHD
}

Prescription trends for stimulants, selective serotonin reuptake inhibitors (SSRI), and combination therapies for ADHD, comorbid and emotional disorders were evaluated by a retrospective population-based analysis of North Carolina Medicaid prescription claim files for the years 1992-1998. In the 7 year study period, prescription prevalence in school-aged children 6 to 14 years increased from $4.4 \%$ to $9.5 \%$ for stimulants, and from $0.2 \%$ to $1.5 \%$ for SSRIs. In preschool children, stimulant prescription prevalence increased from $0.6 \%$ in 1992 to $1.3 \%$ in 1998, and SSRI prevalence from $<0.01 \%$ to $0.1 \%$. Preschool children (aged 1-5 years) accounted for only $7.1 \%$ of all stimulant prescriptions and $2.2 \%$ of SSRI prescription claims for children aged 1-19 years. Combination therapy also increased in prevalence. In 1998, stimulant usage was highest in white male children (18.3\%), compared to $3.4 \%$ in black female children. The respective prevalences for SSRIs were $2.8 \%$ in white males and $0.6 \%$ in black females. Increases were found in number of prescriptions filled, number of patients treated, and in percentage of children prescribed these medications annually. Stimulant usage of almost 10\% in 1998 was greater than the reported ADHD prevalence. (Rushton JL, Whitmire JT. Pediatric stimulant and selective serotonin reuptake inhibitor prescription trends 1992 to 1998. Arch Pediatr Adolesc Med May 2001;155:560-565). (Respond: Jerry L Rushton MD MPH, Department of Pediatrics, University of Michigan, 300 North Ingalls Bldg, Room 6DO5, Ann Arbor, MI 48109).

COMMENT. Annual prescription prevalence of stimulants, SSRIs, and combination therapies in North Carolina school-aged children increased significantly from 1992 through 1998, reaching almost 10\% for stimulants and $1.5 \%$ for SSRIs. Stimulant usage in white children is twice that in black children, and males are medicated atleast twice as often as females. Whereas the higher prevalence of stimulant prescriptions in males may be explained by sex differences in susceptibility to $\mathrm{ADHD}$, the race differences in treatment prevalence are not readily apparent. A greater aversion to stimulant usage among parents of black compared to white children is one possible factor.

\section{IRON DEFICIENCY AND COGNITIVE UNDERACHIEVEMENT}

The relationship between iron deficiency and cognitive test scores among 5398 children, 6 to 16 years old, was studied at the University of Rochester School of Medicine, NY, by analysis of data obtained from the National Health and Nutrition Examination Survey III 1988-1994. Iron deficiency was based on measures of serum ferritin, transferrin saturation, and free erythrocyte protoporphyrin. Standardized test scores were compared for children with normal iron values, iron deficiency without anemia, and iron deficiency with anemia. Among this nationally representative sample of school-aged children and adolescents, 3\% were iron-deficient. Among adolescent girls, the prevalence of iron deficiency was $8.7 \%$ (only $1.5 \%$ with anemia). Average math scores for irondeficient children with and without anemia were 86.4 and 87.4 , respectively, compared to 93.7 for children with normal iron status $(P<.05)$. The block design test score was also significantly lower in iron-deficient children with anemia (8.0 vs $9.5 ; \mathrm{P}<.05)$, and other tests showed a trend toward lower scores. The risk of 
scoring below average in math for iron deficient children was greater than twice that in children with normal iron values. This increased risk was present in both iron-deficient children with or without anemia. (Halterman JS, Kaczorowski JM, Aligne CA, et al. Iron deficiency and cognitive achievement among school-aged children and adolescents in the United States. Pediatrics June 2001;107:1381-1386). (Reprints: Jill S Halterman MD, University of Rochester School of Medicine, 777 Strong Memorial Hospital, 601 Elmwood Ave, Rochester, NY 14642).

COMMENT. Iron-deficiency, with or without anemia, in school-aged children and adolescents is associated with lower scores on standardized math tests. The prevalence of iron deficiency is highest in adolescent girls, reaching $8.7 \%$ in a nationally US representative sample. Screening for iron deficiency should be considered in children with learning and behavior problems, even in those without anemia. A few previous studies have demonstrated adverse effects of anemia on cognitive performance, and variable effects of iron therapy on learning and memory. In future prospective studies, measures of iron status in relation to cognitive test scores appear to be more significant than hemoglobin values.

Reversible focal neurologic deficits were reported in a 14-year-old black female adolescent treated at Duke University Medical Center (Bruggers CS et al. I Pediatr 1990;117:430-432; see Progress in Pediatric Neurology I, PNB Publishers, 1991;pp397-8). After transfusion and treatment with ferrous sulfate, a facial palsy resolved in 12 hours and a VI nerve palsy and somnolence resolved by the fifth day. Despite continued bleeding from hereditary telangiectasia, the neurologic exam remained normal while supplemental iron and a normal hemoglobin were maintained. See Ped Neur Briefs (May 1997;11:33-34) for further reference to iron deficiency and learning disorders.

\section{SEIZURE DISORDERS}

\section{MORTALITY RISK IN CHILDREN WITH EPILEPSY}

The mortality in a cohort of 472 children (aged 1 month to 16 years) with epilepsy, newly diagnosed between 1988 and 1992, was compared in a multicenter study with that in the same age group in the general population in the Netherlands. All children were followed for 5 years or until death. Nine children died, and the mortality rate was $3.8 / 1000$ person-years, seven times higher than expected. Death occurred only among children with epilepsy caused by a static or progressive neurologic disorder ( 6 with neonatal encephalopathy, 3 progressive disorder, 1 Niemann-Pick C, 1 ependymoma, and 1 infantile ceroid lipofuscinosis). None had a sudden unexpected and unexplained death from epilepsy. No mortality occurred among 328 children with epilepsy of nonsymptyomatic cause. In those with symptomatic epilepsy, the mortality risk was 22.9 vs 0.39 expected. (Callenbach PMC, Westendorp RGJ, Geerts AT et al. Mortality risk in children with epilepsy: The Dutch study of epilepsy in childhood. Pediatrics June 2001;107:12591263). (Reprints: Oebele F Brouwer MD PhD, Department of Neurology, University Hospital Groningen, Box 30001, 9700 RB Groningen, The Netherlands).

COMMENT. Compared to the general pediatric population in the Netherlands, the risk of mortality in childhood epilepsy is not increased for nonsymptomatic cases but reaches a 20 -fold increase in children with symptomatic epilepsy. Among the 9 deaths in this prospective study, none was sudden unexpected and unexplained (SUDEP). An accurate initial diagnosis is important in counseling 